severe asthma. We wished to investigate the effects on CANO of the addition of coarse or fine particle inhaled corticosteroids to standard therapy in severe asthma.

Methods Severe asthmatics taking $=1600 \mu \mathrm{g}$ /daybudesonide or equivalent performed a randomised open-label crossover study. Subjects with $\mathrm{FEV}_{1}<80 \%$, gas trapping and elevated CANO $(=2 \mathrm{ppb})$ entered a 6 week dose-ramp run-in of Fluticasone/Salmeterol (FPSM) $250 / 50 \mu \mathrm{g}$ twice daily for 3 weeks, then $500 / 50 \mu \mathrm{g}$ twice daily for 3 weeks. Patients then received additional HFABDP200 $\mu$ twice daily or FP $250 \mu \mathrm{g}$ twice daily for 3 wks in a crossover. Participants then received prednisolone (PRED) $25 \mathrm{mg} /$ day for 1 week. Nitric oxide, lung function, mannitol challenge, systemic inflammatory markers and urinary cortisol were measured.

Results 15 patients completed perprotocol: mean (SD) age, 51 (12) yr; $\mathrm{FEV}_{1}, 58$ (13)\% predicted; residual volume, $193(100) \%$ predicted; mannitol PD10177 (2.8) $\mu \mathrm{g}$. There was no significant difference between FPSM and any add-on therapy for CANO. FPSM/BDP and FPSM/PRED suppressed JawNO and FENO compared to FPSM alone. There was no significant difference between treatments for pulmonary function or bronchial challenge. ECP, e-selectin and ICAM-1 were significantly suppressed by FPSM/ PRED compared to FPSM and FPSM/FP but not FPSM/BDP. Plasma cortisol was significantly suppressed by FPSM/PRED only.

Conclusion In severe asthma, alveolar nitric oxide is insensitive to changes in dose and delivery of inhaled corticosteroids and is not suppressed by systemic corticosteroids. Additional inhaled HFABDP caused reductions in FENO and JawNO without adrenal suppression. Oral prednisolone reduced FENO and JawNO with suppression of systemic inflammatory markers and urinary cortisol.

\section{S10 IS A HIGH FeNO A MARKER OF NON-ADHERENCE IN DIFFICULT ASTHMA?}

doi:10.1136/thoraxjnl-2011-201054b.10

'D M McNicholl, 'L P McGarvey, ${ }^{2} \mathrm{~L}$ G Heaney. ${ }^{1}$ Centre for Infection \& Immunity, Queens University, Belfast, Northern Ireland; ${ }^{2}$ Regional Respiratory Centre, Belfast City Hospital, Belfast, Northern Ireland

Background Non-adherence to inhaled combination therapy (ICT) is a major contributor to poor control in difficult asthma ${ }^{1}$; however identifying non-adherence in this population is challenging. We have shown that suppression of fractional exhaled nitric oxide (FeNO), following directly observed inhaled corticosteroid can identify nonadherence ${ }^{2}$; it has previously been suggested that a single high FeNO measurement can identify non-adherence in difficult asthma.

Methods We performed a retrospective analysis of patients attending a difficult asthma clinic between January 2007 and December 2010. Prescription refill data, patient demographics, FeNO and lung function were collated. ICT prescription refill ratio \% was calculated as the number of doses refilled/number of doses prescribed over 6 months $\times 100 .{ }^{1}$ Non-parametric correlation analysis was performed. Tests for non-adherence defined as ICT prescription refill cut-offs of $<80 \%$ and $<50 \%$ were assessed.

Results One hundred and forty-six patients underwent systematic evaluation during the time period. Patient characteristics are displayed in Abstract S10 table 1. FeNO level and ICT prescription refill ratio did not correlate significantly $(\mathrm{r}=-0.11, \mathrm{p}=0.2)$. For FeNO $>45 \mathrm{ppb}$, there was a trend with ICT prescription refill ratio of $<80 \%(p=0.06)$; significant in patients not prescribed oral steroids $(\mathrm{n}=99, \mathrm{p}=0.016)$. No relationship existed between FeNO $>100 \mathrm{ppb}$ at both prescription refill ratio cut-offs. Using FeNO $>45 \mathrm{ppb}$ to define non-adherence, negative (NPV) and positive predictive values (PPV) were: $66 \%$ and $61 \%$ for $<80 \%$ ICT prescription refill ratio; $71 \%$ and $33 \%$ for $<50 \%$ prescription refill ratio. Using FeNO $>100 \mathrm{ppb}$, NPV and PPV were: $55 \%$ and $52 \%$ for $<80 \%$; $69 \%$ and $32 \%$ for $<50 \%$ prescription refill ratio.

\section{Abstract S10 Table 1 Patient characteristics}

\begin{tabular}{|c|c|}
\hline Patient characteristic & $n=146$ \\
\hline Age (years) $\dagger$ & $40.6 \pm 15.1$ \\
\hline $\operatorname{Sex}(F / M)$ & $100 / 46$ \\
\hline Inhaled corticosteroid (BDP equivalent $\mu \mathrm{g})^{*}$ & $1600(800-2000)$ \\
\hline Long acting $\beta$-agonist, $\mathrm{n}(\%)$ & $142(97)$ \\
\hline Theophylline, $\mathrm{n}(\%)$ & $75(51)$ \\
\hline Maintenance oral steroids, n (\%) & $47(32)$ \\
\hline $\mathrm{FEV}_{1}$ (L) \% predicted $\dagger$ & $79 \pm 24$ \\
\hline $\mathrm{FEV}_{1} / \mathrm{FVC}$ ratio $\% \dagger$ & $69 \pm 13$ \\
\hline FeNO $(p p b)^{*}$ & $46(21-89)$ \\
\hline ICT prescription refill ratio $(\%)^{*}$ & $89(49-103)$ \\
\hline
\end{tabular}

*Median (IQR).

†Mean \pm SD.

Conclusion While there is a weak relationship between a single point-in-time FeNO measurement and non-adherence to ICT in difficult asthma, this cannot be used as an alternative to our previously described FeNO suppression test.

\section{REFERENCES}

1. Gamble, et al. AJRCCM 2010;180:817-22.

2. McNicholl, et al. AJRCCM 2011;183:A437.

\section{S11 INAPPROPRIATE PRESCRIBING OF COMBINATION INHALERS IN ASTHMA IN NORTHERN IRELAND (NI)}

doi:10.1136/thoraxjnl-2011-201054b.11

${ }^{1} \mathrm{~J}$ Sweeney, ${ }^{2} \mathrm{~A}$ M Marley, ${ }^{3} \mathrm{C}$ Patterson, ${ }^{1} \mathrm{~L}$ G Heaney. ${ }^{1}$ Centre for Infection and Immunity, Queens University, Belfast, UK; ${ }^{2}$ Belfast Health and Social Care Trust, Belfast, UK; ${ }^{3}$ Centre for Public Health, Queens University, Belfast, UK

Introduction BTS/SIGN asthma management guidelines recommend a stepwise approach to the use of anti-inflammatory therapy, including the addition of inhaled combination therapy (ICT) at Step 3. In NI, ICT accounts for $47 \%$ ( $£ 23$ million) of the entire respiratory drug spend suggesting excessive utilisation of ICT.

Methods Using data from a large representative sample of GP practices in NI, we looked at subjects who had a new prescription of ICT (Symbicort, Seretide, Fostair). Data were collected from the Information and Registration Unit of the Business Services Organisation for the period January to December 2010 inclusive for subjects aged 5 to 35 . We examined treatment prior to ICT, SABA, oral steroid and antibiotic use and, prescription filling in the preceding 6 and 12 months.

Results 42665 subjects received 2 or more prescriptions for any respiratory drug (BNF categories 3.1, 3.2, 3.3) and of these 12989 received ICT with 3953 new ICT prescriptions. 2642 (67\%) of these had no ICS in study year or 6 month lead in period. A further analysis of a 12-month lead in period showed 39315 subjects with 2 or more respiratory prescriptions and out of these 11962 received ICT with 2609 new ICT prescriptions. 1359 (52\%) had no ICS in the study year or 12 -month lead in. A sub-group analysis $(n=600)$ showed that $51 \%$ of first prescriptions for ICT are made in Jan-April but in the previous 6 months only $23 \%$ are issued a SABA, $5 \%$ receive OCS and $31 \%$ receive an antibiotic.

Conclusion ICT is initiated in the majority of young asthmatic subjects without prior inhaled steroid therapy. Most prescriptions are initiated in the January-April period and do not appear to be driven by severe asthma exacerbation (oral steroid prescription) or worsening asthma control (SABA use). Significant reductions in ICT, with associated cost savings, would occur if the BTS/SIGN prescribing guidelines were followed in primary care. We are currently trying to identify the drivers and potential economic impact of poor adherence to national prescribing guidelines and 
examine ICT prescribing in other UK regions to identify if this is a more widespread problem.

\section{S12 UNSCHEDULED HEALTHCARE RESOURCE UTILISATION AND HEALTH-RELATED QUALITY OF LIFE BEFORE AND AFTER OMALIZUMAB INITIATION IN UK CLINICAL PRACTICE: THE APEX STUDY}

doi:10.1136/thoraxjnl-2011-201054b.12

\begin{abstract}
${ }^{1} \mathrm{~N}$ Barnes, ${ }^{2} \mathrm{~A}$ Radwan, ${ }^{3} \mathrm{~F}$ Percival. ${ }^{1}$ Barts and The London NHS Trust, London, UK; ${ }^{2}$ Novartis Pharmaceuticals UK Limited, Frimley, UK; ${ }^{3} \mathrm{pH}$ Associates Limited, Marlow, $U K$
\end{abstract}

Objectives The efficacy and safety of omalizumab for the treatment of severe persistent allergic asthma have been demonstrated in randomised controlled clinical trials. However, there are limited "real world" data on its effects on healthcare resource utilisation or health-related quality of life (QoL) in UK clinical practice.

Methods A 10 centre retrospective observational study (APEX) compared 12 months pre-vs 12 months post-omalizumab initiation in patients aged $=12$ years with severe persistent allergic asthma. All patients received $=1$ dose of omalizumab. Patients who had received omalizumab in a clinical trial were excluded. Hospital records were reviewed to obtain data on hospital resource use and routinely used QoL measures for example, Asthma Quality of Life Questionnaire (AOLO) at baseline (pre-omalizumab), 16 weeks and up to 12 months following omalizumab initiation.

Results Mean in-patient hospital admissions fell by $61 \%$ from 1.30 to $0.51(\mathrm{p}<0.001)$ and mean in-patient bed days fell by $70 \%$ from 9.10 to $2.74(p<0.001)$ per patient. In the subgroup of patients hospitalised for asthma in the 12 months pre-omalizumab $(n=81)$, mean in-patient hospital admissions fell by $70 \%$ from 2.19 to 0.65 $(p<0.001)$ and mean in-patient bed days fell by $74 \%$ from 14.86 to $3.83(p<0.001)$ per patient. Similarly, mean Accident and Emergency department attendances fell by $70 \%$ from 1.52 per patient in the 12 months pre-omalizumab to 0.46 in the 12 months post-omalizumab $(p<0.001)$. Other resource use, such as outpatient attendances (excluding visits made solely for omalizumab administration), nurse appointments and telephone consultations remained unchanged following omalizumab initiation. OoL data were not available for all patients at every time point. However, where data were available, mean AOLO scores increased from 3.09 at baseline to 5.01 at 16 weeks $(n=90)$ and to 5.22 at 12 months $(n=29)$.

Conclusions Treatment with omalizumab is associated with a clinically and statistically significant reduction in unplanned hospital resource utilisation and improvements in patients' QoL.

\section{Unravelling airway infection in COPD S13 DIFFERENTIATED HUMAN RHINOVIRUS LOADS IN STABLE
COPD AND AT EXACERBATION}

doi:10.1136/thoraxjnl-2011-201054b.13

S N George, A J Mackay, A R C Patel, R J Sapsford, D S Garcha, G C Donaldson, J A Wedzicha. University College London, London, UK

Introduction Human rhinoviruses (HRV) are the main aetiological agents of virus-associated COPD exacerbations (Seemungal et al, 2001). However the importance of the level of viral load as a trigger for naturally occurring exacerbations is not fully understood. We aimed to assess and quantify HRV prevalence and load in stable and exacerbating patients from the London COPD cohort.

Methods A real-time qPCR protocol was utilised to detect HRV presence and quantify load in sputum samples taken at baseline $(n=58)$ and at COPD exacerbation onset $(n=57)$. COPD patients were defined as stable if exacerbation-free for at least 42 days since the previous exacerbation and more than 14 days before the next. Exacerbations were defined using our usual symptom criteria; an increase in respiratory symptoms for two consecutive days, with at least one symptom being a major symptom; dyspnoea, sputum purulence or volume and the other a major or minor symptom; wheeze, cold sore throat, and cough (Anthonisen criteria). A $\chi^{2}$ test was used to compare HRV prevalence of the two disease phases, and an independent-samples $t$ test was used to compare the differences in viral load.

Results Sixty-four patients provided 115 sputum samples: mean age 70.5 years ( $\mathrm{SD} \pm 8.1) ; \mathrm{FEV}_{1} 45.8 \%$ predicted $( \pm 20.0 \%)$; current smoker $31.3 \%$. There is a significantly higher prevalence of HRV at exacerbation onset, $31.6 \%(18 / 57)$ compared to baseline $15.5 \%$ (9/ 58) $(\mathrm{p}=0.042)$. Similarly, the mean viral load was significantly greater at exacerbation onset $1.70( \pm 1.67) \log _{10} \mathrm{pfu} / \mathrm{ml}$ than baseline $0.30( \pm 0.69) \quad \log _{10} \mathrm{pfu} / \mathrm{ml}(\mathrm{p}=0.025)$, exhibiting a 25 -fold increase in viral load from baseline to exacerbation. $6.9 \%(4 / 58)$ of patients were positive for HRV at baseline only, 26.3\% (15/57) at exacerbation only and $8.8 \%(4 / 45)$ at both time points $(p=0.006)$.

Conclusions HRV load is significantly greater at COPD exacerbation than when detected in the stable state. This emphasises the importance of HRV as a key trigger of COPD exacerbations. HRV can be detected in the stable state; however the loads are very low suggesting asymptomatic carriage rather than chronic infection.

\section{S14 HISTONE DEACETYLASE ACTIVITY IS REDUCED IN COPD SUBJECTS DURING RHINOVIRUS INDUCED EXACERBATIONS}

doi:10.1136/thoraxjnl-2011-201054b.14

J I M Footitt, P Mallia, M B Trujillo-Torralbo, A Durham, I M Adcock, S L Johnston. NHLI, Imperial College, London, UK

Introduction and Objectives Histone deacetylase (HDAC) enzymes have a role in suppressing inflammatory gene transcription. There is evidence for reduced HDAC activity in COPD which correlates with the severity of airflow obstruction. Increased inflammation found during exacerbations of COPD may result from a reduction in HDAC activity but this has not been studied in virus induced exacerbations. We sought to investigate HDAC activity following rhinovirus infection in an experimental model of COPD exacerbations.

Methods Experimental rhinovirus challenge was performed in GOLD stage II COPD subjects and non-obstructed control smokers and non-smokers. Rhinovirus infection was confirmed with quantitative PCR performed on nasal lavage and sputum samples collected at baseline and days 3, 5, 9, 12, 15, 21 and 42 post virus inoculation. Sputum macrophages were isolated by adhesion and HDAC2 isoenzyme immunoprecipitated, prior to performing a HDAC activity assay.

Results 11 non-smokers (NS), 10 smokers (Smk) and 9 COPD subjects were recruited. The mean (SEM) \% predicted baseline $\mathrm{FEV}_{1}$ was 103 (4), 98 (4) and 69 (2)\% for NS, Smk and COPD respectively. At baseline there was no difference in HDAC2 activity between the three study groups, the geometric mean $(95 \% \mathrm{CI})$ activity was NS 22.39 (12.45 to 40.25); Smk 22.91 (18.28 to 28.71) and COPD 48.98 (26.04 to 92.13 ) ( $p=0.095)$. Following rhinovirus infection, in NS HDAC2 activity increased, in Smk it remained largely unchanged and there was a fall from baseline levels in COPD subjects at all time points, Abstract S14 figure 1.

Conclusions HDAC2 activity was not reduced in stable COPD subjects compared to controls. This may reflect the mild disease state of the study population. During a rhinovirus induced 